\title{
Novel "Add-On" Molecule Based on Evans Blue Confers Superior Pharmacokinetics and Transforms Drugs to Theranostic Agents
}

\author{
Haojun Chen*1,2, Orit Jacobson*2, Gang $\mathrm{Niu}^{2}$, Ido D. Weiss ${ }^{3}$, Dale O. Kiesewetter ${ }^{2}$, Yi Liu ${ }^{2}$, Ying Ma², Hua Wu ${ }^{1}$, \\ and Xiaoyuan Chen ${ }^{2}$ \\ ${ }^{1}$ Department of Nuclear Medicine, Xiamen Cancer Hospital of the First Affiliated Hospital of Xiamen University, Xiamen, China; \\ ${ }^{2}$ Laboratory of Molecular Imaging and Nanomedicine, National Institute of Biomedical Imaging and Bioengineering, National \\ Institutes of Health, Bethesda, Maryland; and ${ }^{3}$ Laboratory of Molecular Immunology, National Institute of Allergy and Infectious \\ Diseases, National Institutes of Health, Bethesda, Maryland
}

One of the major design considerations for a drug is its pharmacokinetics in the blood. A drug with a short half-life in the blood is less available at a target organ. Such a limitation dictates treatment with either high doses or more frequent doses, both of which may increase the likelihood of undesirable side effects. To address the need for additional methods to improve the blood half-life of drugs and molecular imaging agents, we developed an "add-on" molecule that contains 3 groups: a truncated Evans blue dye molecule that binds to albumin with a low micromolar affinity and provides a prolonged half-life in the blood; a metal chelate that allows radiolabeling for imaging and radiotherapy; and maleimide for easy conjugation to drug molecules. Methods: The truncated Evans blue molecule was conjugated with the chelator NOTA or DOTA, and the resulting conjugate was denoted as NMEB or DMEB, respectively. As a proof of concept, we coupled NMEB and DMEB to $\mathrm{c}(\mathrm{RGDfK})$, which is a small cyclic arginine-glycine-aspartic acid (RGD) peptide, for targeting integrin $\alpha_{v} \beta_{3}$. NMEB and DMEB were radiolabeled with ${ }^{64} \mathrm{Cu}$ and ${ }^{90} \mathrm{Y}$, respectively, and tested in xenograft models. Results: The resulting radiolabeled conjugates showed a prolonged circulation half-life and enhanced tumor accumulation in integrin $\alpha_{v} \beta_{3}$-expressing tumors. Tumor uptake was markedly improved over that with NOTA- or DOTA-conjugated c(RGDfK). Tumor radiotherapy experiments in mice with ${ }^{90} \mathrm{Y}-\mathrm{DMEB}-\mathrm{RGD}$ showed promising results; existing tumors were eliminated. Conclusion: Conjugation of our novel add-on molecule, NMEB or DMEB, to potential tracers or therapeutic agents improved blood half-life and tumor uptake and could transform such agents into theranostic entities.

Key Words: Evans blue; albumin binding; RGD peptide; integrin; theranostics; ${ }^{90} \mathrm{Y}$

J Nucl Med 2017; 58:590-597

DOI: 10.2967/jnumed.116.182097

Received Aug. 2, 2016; revision accepted Nov. 9, 2016.

For correspondence or reprints contact: Xiaoyuan Chen, Laboratory of Molecular Imaging and Nanomedicine, National Institute of Biomedical Imaging and Bioengineering, National Institutes of Health, 35A Convent Dr., GD937, Bethesda, MD 20892.

E-mail: shawn.chen@nih.gov

${ }^{*}$ Contributed equally to this work.

Published online Nov. 22, 2016.

COPYRIGHT (C) 2017 by the Society of Nuclear Medicine and Molecular Imaging.
$\mathbf{T}$ he goal of drug development is to achieve high activity and specificity for a desired biologic target. However, many potential pharmaceuticals that meet these criteria fail as therapeutics because of unfavorable pharmacokinetics, in particular, rapid blood clearance, which prevents the achievement of therapeutic concentrations. For some drugs, the administration of large or frequently repeated doses is required to achieve and maintain therapeutic levels (1) but can, in turn, increase the probability of undesired side effects. One chemical modification that delays the rate of drug clearance and has been effective for some drugs is the addition of polyethylene glycol (PEG) (2). However, recent studies showed that PEGylation of drugs has disadvantages, including immunogenicity caused by the development of anti-PEG antibodies, heterogeneity of the PEGylated drugs, and decreased biologic activity and bioavailability of the drugs $(1,3)$.

To address the need for additional methods to improve the blood half-life of drugs and molecular imaging agents, we made use of molecules with a high affinity for albumin. We previously developed a radiolabeled truncated derivative of Evans blue (EB) that allowed imaging of the blood volume because of the affinity of EB for albumin (4). This result encouraged us to explore drug conjugates of EB derivatives that would use albumin as a reversible carrier for drug delivery. We hypothesized that the micromolar affinity and reversible binding of EB derivatives to albumin would extend the half-life of a drug in blood. Additionally, slow release of the drug over time would allow continual uptake at the biologic target. We demonstrated this enhanced blood half-life and drug efficacy for a derivative of exendin-4 $(5,6)$.

To adapt this approach to theranostic applications, we now report a novel molecule that contains the same truncated EB molecule, a metal chelate, and maleimide and allows conjugation to a drug or targeting moiety through appropriate chemistry (Fig. 1). We prepared analogs with NOTA or DOTA as the chelator (NMEB or DMEB, respectively). As a proof of concept, we conjugated NMEB and DMEB to $\mathrm{c}$ (RGDfK), hereafter referred to as arginine-glycine-aspartic acid (RGD) peptide, for targeting cell surface receptor integrin $\alpha_{v} \beta_{3}$. This integrin receptor is overexpressed in various malignancies and has a crucial role in tumor angiogenesis (7-9). We demonstrated that either NMEB-RGD or DMEB-RGD could be of use as an imaging agent and as a radiotherapeutic agent for the treatment of integrin $\alpha_{\mathrm{v}} \beta_{3}-$ expressing tumors. The use of these novel EB derivatives confers the potential transformation of many other drugs into theranostic agents. 


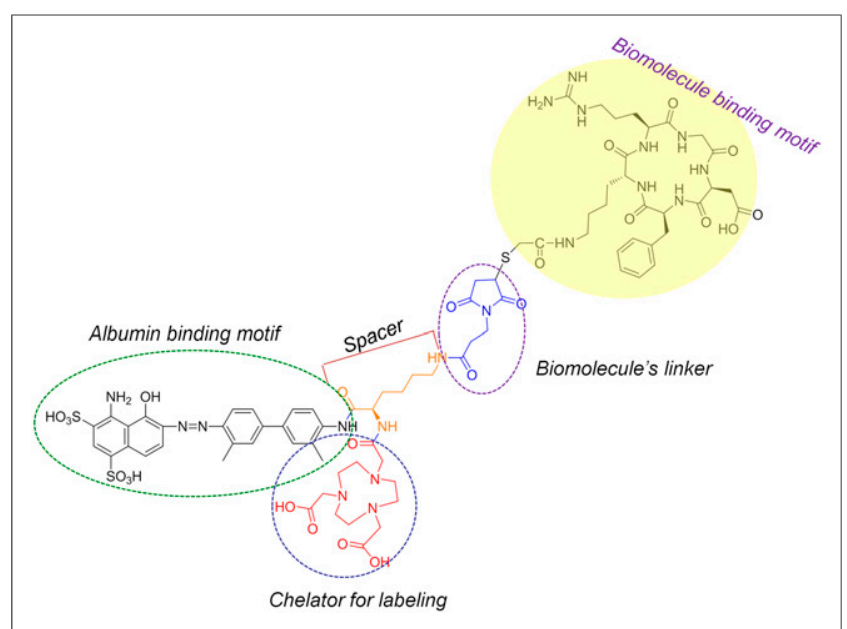

FIGURE 1. Chemical structure of NMEB-RGD.

\section{MATERIALS AND METHODS}

\section{General}

Boc-lysine-Fmoc amino acid was purchased from Bachem. NOTA-bis ( $t$-butyl ester) and DOTA-tris( $t$-butyl ester) were purchased from Macrocyclics. $N$-Succinimidyl $S$-acetylthioacetate and the $\mathrm{PEG}_{4}$ biotinylation reagent were purchased from Thermo Fisher Scientific. Avidin-Sepharose beads were acquired from GE Healthcare. Cyclic-(Arg-Gly-Asp-D-Phe-Lys) (RGD) peptide was purchased from C.S. Bio. All other solvents and chemicals were purchased from Sigma-Aldrich.

Analytic high-performance liquid chromatography (HPLC) was done on a Phenomenex Luna C8 column $(5 \mu \mathrm{m} ; 4.60 \times 150 \mathrm{~mm})$ with 2 gradient systems. System 1 was a gradient starting from $80 \%$ solvent A $\left(50 \mathrm{mM} \mathrm{NH}_{4} \mathrm{OAc}\right)$ and $20 \%$ solvent $\mathrm{B}\left(\mathrm{CH}_{3} \mathrm{CN}\right)$ for $2 \mathrm{~min}$ and increasing to $90 \%$ solvent B over $15 \mathrm{~min}$ at a flow rate of $1 \mathrm{~mL} / \mathrm{min}$. System 2 was a gradient starting from $95 \%$ solvent $\mathrm{A}$ and $5 \%$ solvent $\mathrm{B}$ and changing to $65 \%$ solvent $B$ over $35 \mathrm{~min}$ at a flow rate of $1 \mathrm{~mL} / \mathrm{min}$. The ultraviolet absorbance was monitored at 254 and $600 \mathrm{~nm}$. Compounds were purified on either a Biotage purification system (C-18; $210 \times 25 \mathrm{~mm})$ or a Higgins column $(\mathrm{C}-18 ; 5 \mu \mathrm{m} ; 250 \times 20 \mathrm{~mm})$ with gradient system 2 and a flow rate of 25 or $12 \mathrm{~mL} / \mathrm{min}$, respectively; the gradient was similar to that of system 2 except for a change in the solvents (solvent A: $0.1 \%$ trifluoroacetic acid and $\mathrm{H}_{2} \mathrm{O}$; solvent B: $0.1 \%$ trifluoroacetic acid and $\mathrm{CH}_{3} \mathrm{CN}$ ).

Liquid chromatography-mass spectrometry (LC-MS) analysis was done in a manner similar to the reported procedure $(4) .{ }^{64} \mathrm{CuCl}_{2}$ was acquired from the National Institutes of Health Cyclotron Facility. Radio-thin-layer chromatography was done on a Bioscan AR-2000 scanner with instant TLC plates and $0.1 \mathrm{M}$ citric acid (pH 5) as a developing solvent. ${ }^{18} \mathrm{~F}-\mathrm{FGD}$ was purchased from Cardinal Health. $3^{\prime}$-Deoxy- $3^{\prime}-{ }^{18} \mathrm{~F}-$ fluorothymidine $\left({ }^{18} \mathrm{~F}-\mathrm{FLT}\right)$ was synthesized in accordance with the known procedure (10).

\section{Chemistry}

Synthesis of EB-Lys-Boc. EB- $\mathrm{NH}_{2}$ was synthesized as described previously (4). To a solution of Boc-Lys-Fmoc (80-100 mM; 3.6 equivalents [Eq]) in anhydrous $N, N$-dimethylformamide (DMF) was added (1-[bis (dimethylamino)methylene]-1H-1,2,3-triazolo[4,5-b]pyridinium 3-oxide hexafluorophosphate) (HATU; 4.2 Eq) as a solid under argon. The solution was stirred for $10 \mathrm{~min}$ at room temperature (RT). Next, $10 \mathrm{Eq}$ of diisopropylethylamine were added, and then $\mathrm{EB}^{-\mathrm{NH}_{2}}(11-17 \mathrm{mM} ; 1 \mathrm{Eq})$ in DMF was added. The progress of the reaction was monitored by analytic HPLC with system 1 . The reaction mixture was stirred overnight at RT. The retention time of EB-NH $\mathrm{N}_{2}$ was $7.7 \mathrm{~min}$, and that of conjugated EB-protected Lys was $11 \mathrm{~min}$. After the reaction mixture was stirred overnight, HPLC indicated complete conversion. Piperidine (20\% [v/v]) was added, and the reaction mixture was stirred for $1 \mathrm{~h}$ to cleave the Fmoc-protecting group. DMF was removed under high vacuum, and the residue was redissolved in methanol- $\mathrm{H}_{2} \mathrm{O}(2: 1)$ and purified on a Biotage system. The collected fractions were reinjected into an analytic HPLC system, and those displaying a purity of greater than $90 \%$ were combined and lyophilized to provide the product in an isolated yield of $40 \%$. The EB-Lys-Boc retention time was $8.3 \mathrm{~min}$ (system 1) or $23.2 \mathrm{~min}$ (system 2). LC-MS analysis confirmed a mass of $769\left([\mathrm{M}-\mathrm{H}]^{-}\right.$.

Synthesis of NOTA-EB-Lys-Boc. The reaction between EB-Lys-Boc and NOTA-bis( $t$-butyl ester) was done under conditions similar to those described earlier in the presence of $4.2 \mathrm{Eq}$ of HATU and $10 \mathrm{Eq}$ of base. Analytic HPLC system 2 confirmed a purity of greater than $90 \%$; the retention time was $29.3 \mathrm{~min}$, the mass was $1,167\left([\mathrm{M}-\mathrm{H}]^{-}\right)$, and the isolated yield was $65 \%$.

Synthesis of NOTA-EB-Lys. Deprotection was done at RT with thioanisole-1,2-ethanedithiol-anisole-trifluoroacetic acid (5:3:2:90) in a total volume of $2 \mathrm{~mL}$. Completion of deprotection was monitored by HPLC (retention time, $17.1 \mathrm{~min}$ ). Trifluoroacetic acid was removed by argon flow before purification. NOTA-EB-Lys was purified on a Biotage system. LC-MS analysis confirmed a mass of $954\left([\mathrm{M}-\mathrm{H}]^{-}\right)$; the isolated yield was $88 \%$.

Synthesis of NOTA-Maleimide-EB (NMEB). NOTA-EB-Lys (10$14 \mathrm{mM}$ ) was dissolved in DMF. Next, $1.26 \mathrm{Eq}$ of triethylamine and $1.26 \mathrm{Eq}$ of 3-(maleimido)propionic acid $N$-hydroxysuccinimide ester were added in succession. The reaction mixture was stirred for $2 \mathrm{~h}$ at RT. Purification was done on a Higgins column. Analytic HPLC system 2 revealed a purity of greater than $90 \%$; the retention time was 17.4 min, the mass was $1,105\left([\mathrm{M}-\mathrm{H}]^{-}\right)$, and the isolated yield was $60 \%$.

Synthesis of Thiolated RGD. RGD (20-30 mg) was dissolved in $1.5 \mathrm{~mL}$ of $(100 \mathrm{mM}) \mathrm{Na}_{2} \mathrm{HPO}_{4}(\mathrm{pH}$ 7.5). $\mathrm{N}$-Succinimidyl $S$-acetylthioacetate (1.3 Eq) was dissolved in dimethyl sulfoxide and added to the peptide solution. The solution was stirred for 1-1.5 h until HPLC showed complete conversion to the conjugated peptide. The solvent was removed by lyophilization overnight. Deprotection of the acetyl group was done with $70 \mathrm{mg}$ of hydroxylamine and $20 \mathrm{mg}$ of ethylenediaminetetraacetic acid in $0.1 \mathrm{M}$ borate buffer $\left(\mathrm{pH}\right.$ 8.6): $\mathrm{H}_{2} \mathrm{O}$ (1:1) (in a total volume of 3 $\mathrm{mL}$ ) for $1 \mathrm{~h}$ at RT. Purification of thiolated RGD was done on a Higgins column. Reinjection of the pure peptide into an analytic HPLC system revealed a purity of greater than $90 \%$; the retention time was $17.3 \mathrm{~min}$. LC-MS analysis confirmed a mass of $676\left([\mathrm{M}-\mathrm{H}]^{-}\right)$.

Synthesis of NMEB-RGD. NMEB $(15-20 \mathrm{mM})$ was dissolved in $0.3 \mathrm{~mL}$ of degassed $0.1 \%$ sodium ascorbate (w/v) in phosphate-buffered saline (PBS). Thiolated RGD (1.1 Eq) was dissolved in $50 \mu \mathrm{L}$ of DMF, and this mixture was added to the NMEB solution. The reaction mixture was stirred at RT for $2 \mathrm{~h}$. Purification was done on a Higgins system. The NMEB-RGD retention time was $17.54 \mathrm{~min}$, the chemical purity was greater than $90 \%$, and the mass was $1,783\left([\mathrm{M}-\mathrm{H}]^{-}\right)$.

The synthesis of DMEB was conducted in the same manner but with DOTA-tris(t-butyl ester) as a chelator; the HPLC retention time was $17.8 \mathrm{~min}$, and the mass was $1,883.9\left([\mathrm{M}-\mathrm{H}]^{-}\right)$.

\section{Radiochemistry}

For the labeling of NMEB-RGD, $10 \mu \mathrm{L}$ of ${ }^{64} \mathrm{CuCl}_{2}(1.5-2.2 \mathrm{GBq}$; $42-60 \mathrm{mCi}$ ) were diluted with $0.5 \mathrm{~mL}$ of $0.4 \mathrm{M}$ ammonium acetate (pH 5.6). Next, $0.37-0.74 \mathrm{GBq}(10-20 \mathrm{mCi})$ were transferred into a vial containing the peptide $(100 \mu \mathrm{g})$. The reaction contents were mixed for $30 \mathrm{~min}$ at $37^{\circ} \mathrm{C}$ and then tested for purity with either analytic HPLC system 1 (retention time, 6.37) or radio-thin-layer chromatography. The $\mathrm{R}_{\mathrm{f}}$ of free ${ }^{64} \mathrm{Cu}$ was approximately 0.9 , and the $\mathrm{R}_{\mathrm{f}}$ of ${ }^{64} \mathrm{Cu}-\mathrm{NMEB}-\mathrm{RGD}$ was approximately 0.1 . RGD and NEB (the albumin-binding moiety) were labeled with ${ }^{64} \mathrm{Cu}$ in the same manner. Labeling with $370-444$ 
$\mathrm{mBq}(10-12 \mathrm{mCi})$ of ${ }^{90} \mathrm{Y}$ (Perkin-Elmer) was done under conditions similar to those described for ${ }^{64} \mathrm{Cu}$.

\section{Evaluation in Vitro}

Cell Culture. U87MG human glioblastoma, MDA-MB-435 human melanoma, and HT-29 human colorectal adenocarcinoma cell lines were purchased from the American Type Culture Collection and grown as recommended by the American Type Culture Collection.

Measurement of Binding Affinity with Cell-Binding Assays. NMEBRGD cell-binding assays were performed with U87MG human glioblastoma cells in a 96-well membrane plate. Incubation was conducted for $4 \mathrm{~h}$ at RT with a $200-\mu \mathrm{L}$ mixture containing $10^{5}$ cells suspended in serum-free medium or $1 \%(\mathrm{w} / \mathrm{v})$ human serum albumin (HSA), 0.0185 $\mathrm{MBq}$ of ${ }^{64} \mathrm{Cu}-\mathrm{RGD}$, and various concentrations of RGD or NMEB-RGD $(0-5,000 \mathrm{nM})$. After the incubation, the plate was washed 3 times with PBS. The radioactivity in each well was measured with a $\gamma$-counter. Inhibitory concentrations of $50 \%\left(\mathrm{IC}_{50} \mathrm{~s}\right)$ were calculated by fitting the data by nonlinear regression with GraphPad Prism. Experiments were performed in quadruplicate.

Binding assays were done under conditions that were suboptimal because ${ }^{64} \mathrm{Cu}$-RGD does not have a high specific activity, and some of the receptors might have been blocked. However, no other integrin $\alpha_{v} \beta_{3}$ radioligand is commercially available.

The in vitro albumin-binding affinity of NMEB-RGD was assessed via competition with ${ }^{64} \mathrm{Cu}-\mathrm{NMEB}-\mathrm{RGD}$ on avidin-Sepharose beads and biotinylated albumin. Biotinylation of human albumin was done with the EZ-link $\mathrm{PEG}_{4}$ biotinylation reagent in accordance with the manufacturer's instructions. In brief, the beads were incubated with biotinalbumin for $1 \mathrm{~h}$ and then extensively washed. The beads were incubated with ${ }^{64} \mathrm{Cu}$-NMEB-RGD and increasing concentrations $(0.1 \mathrm{nM}-10 \mu \mathrm{M})$ of unlabeled NMEB-RGD for $1 \mathrm{~h}$ and then extensively washed with binding buffer. Thereafter, the radioactivity bound to the beads was measured with a $\gamma$-counter.

Cell Uptake and Internalization. For cell uptake and internalization studies, U87MG, MDA-MB-435, and HT-29 cells were seeded in 24well plates at a density of $10^{5}$ cells per well $24 \mathrm{~h}$ before the assays. The medium was removed, and the cells were rinsed twice with PBS. Next, $18.5 \mathrm{mBq}(0.5 \mu \mathrm{Ci})$ of ${ }^{64} \mathrm{Cu}-\mathrm{NMEB}-\mathrm{RGD}$ in $0.5 \mathrm{~mL}$ of serum-free medium or medium containing $1 \%$ HSA were added per well. The cells were incubated at $37^{\circ} \mathrm{C}$ for $5,30,60$, and $240 \mathrm{~min}$. At each time point, the medium was removed, and the cells were rinsed twice with $1 \mathrm{~mL}$ of PBS and lysed by the addition of $0.2 \mathrm{~mL}$ of $0.1 \mathrm{M} \mathrm{NaOH}$.

For internalization studies, after the removal of the medium at the indicated time points, the cells were incubated for 1 min with $0.5 \mathrm{~mL}$ of acid buffer (50 mM glycine and $100 \mathrm{mM} \mathrm{NaCl}$; pH 2.8). Next, the acid buffer was removed, the cells were washed twice with $1 \mathrm{~mL}$ of PBS, and $0.1 \mathrm{M} \mathrm{NaOH}$ was added. Cell lysates were collected, and the radioactivity was measured with a $\gamma$-counter. The cell uptake and internalization values were normalized to the amount of added radioactivity. Each experiment was repeated twice in triplicate.

Stability in Mouse Serum. ${ }^{64} \mathrm{Cu}-\mathrm{NMEB}-\mathrm{RGD}$ (7.4-11.1 MBq [0.2-0.3 $\mathrm{mCi}$ ) was incubated with $0.5 \mathrm{~mL}$ of mouse serum for $1,4,8$, and $24 \mathrm{~h}$ at $37^{\circ} \mathrm{C}$. At each time point, an aliquot was analyzed by radio-thin-layer chromatography to assess radiochemical stability.

Cell Uptake of NMEB-RGD and Fluorescein Isothiocyanate-Albumin. Cells $\left(10^{5}\right)$ in 24-well plates were incubated with a constant amount of fluorescein isothiocyanate-albumin and increasing amounts of NMEBRGD for $2 \mathrm{~h}$. Next, the cells were washed with PBS and analyzed with an LSR II flow cytometer (BD Biosciences). Cell fluorescence was expressed as mean fluorescence intensity. All cell uptake experiments were conducted in triplicate.

Histopathologic Staining. For the visualization of endothelial cells, integrin expression on tumor vasculature, and integrin expression of tumor cells, immunofluorescence staining with CD31, CD61 (murine integrin $\beta_{3}$ ), and human integrin $\alpha_{v} \beta_{3}$, respectively, was chosen. The mouse antihuman integrin $\alpha_{v} \beta_{3}$ antibody recognizes human integrin $\alpha_{\mathrm{v}} \beta_{3}$ and does not cross-react with murine integrin $\alpha_{\mathrm{v}} \beta_{3}$ on tumor cells. In brief, frozen tissue sections $(5 \mu \mathrm{m})$ were fixed with cold acetone and rinsed with PBS; blocking was done with $1 \%$ bovine serum albumin solution for $1 \mathrm{~h}$ at RT. The slides were incubated with a 1:100 dilution of rat antimouse CD31, hamster antirat CD61, or mouse antihuman integ$\operatorname{rin} \alpha_{\mathrm{v}} \beta_{3}$ monoclonal antibody at room temperature overnight and then incubated with 1:200 Alexa Fluor 488-labeled donkey antirat, Alexa Fluor 647-labeled antihamster, or Alexa Fluor 488-labeled antimouse secondary antibody, respectively. Samples were mounted with $4^{\prime}$,6-diamidino-2-phenylindole (DAPI; Vector) for staining of cell nuclei. Fluorescence images were acquired with an epifluorescence microscope (200×; X81; Olympus). Images were acquired under the same conditions and displayed at the same scale.

Histopathologic Staining After Targeted Radiotherapy. U87MG tumor samples from 6 groups of mice (groups A-F) were collected and sectioned. The CD31 staining procedure was the same as that described earlier. For Ki-67 staining, frozen tumor sections were fixed with cold acetone for $20 \mathrm{~min}$ and dried in the air for $30 \mathrm{~min}$ at RT. After blocking was done with $1 \%$ bovine serum albumin for $30 \mathrm{~min}$, the slides were stained with $\mathrm{Ki}-67-$ specific monoclonal antibody (1:1,000; Abcam) and then incubated with Cy-3-conjugated donkey antirabbit secondary antibody (1:200; Thermo Fisher Scientific). After being washed 3 times with PBS, the samples were mounted with DAPI for staining of cell nuclei. Fluorescence images were acquired with an epifluorescence microscope (200x; X81; Olympus).

Immunofluorescent terminal deoxynucleotidyl transferase-mediated dUTP-biotin nick-end labeling (TUNEL) analysis was done by use of a commercially available kit (Roche Applied Science). In accordance with the manufacturer's specifications, samples were fixed with $10 \%$ formalin and permeabilized by incubation with $0.1 \%$ Triton for 2 min on ice. After fixation and permeabilization, $50 \mu \mathrm{L}$ of TUNEL reaction mixture were added to the samples. The slides were incubated in a humidified atmosphere for $60 \mathrm{~min}$ at $37^{\circ} \mathrm{C}$ in the dark. After being rinsed with PBS and mounted with DAPI, the samples were observed under an epifluorescence microscope with the green fluorescent protein channel. Staining of human tissue was done by Histoserv, Inc., with antibody sc-7312 (Santa Cruz Biotechnology, Inc.). Hematoxylin and eosin staining of mouse tissues was also conducted by Histoserv, Inc.

\section{Evaluation in Vivo}

Stability Assays in FVB Mice. FVB mice were injected with $3.7 \mathrm{MBq}$ $(100 \mu \mathrm{Ci})$ of ${ }^{64} \mathrm{Cu}-\mathrm{NMEB}-\mathrm{RGD}$. At 1 and $4 \mathrm{~h}$, mice $(n=2)$ were euthanized, and blood was withdrawn from the heart. The red blood cells were separated from the plasma by centrifugation $(1,200 \mathrm{~g}$ for $5 \mathrm{~min}$ ). Next, $0.2 \mathrm{~mL}$ of the plasma was diluted with cold methanol (1:1), stirred with a vortex mixer for $30 \mathrm{~s}$, and centrifuged for $5 \mathrm{~min}$ at $10,600 \mathrm{~g}$. The extracted supernatant was filtered through a $0.45-\mu \mathrm{m}$ filter and injected into analytic HPLC system 1.

Tumor Model. Female athymic nude mice (Harlan Laboratories) were housed in an animal facility under pathogen-free conditions. The tumor model was developed in 5- to 6-wk-old mice by injection of $5 \times 10^{6}$ cells into their right shoulders. The mice received ${ }^{90} \mathrm{Y}$ radionuclide therapy when the tumor volume reached $150 \mathrm{~mm}^{3}(10-14 \mathrm{~d}$ after inoculation) and underwent small-animal PET studies when the tumor volume reached $300 \mathrm{~mm}^{3}$ (14-20 d after inoculation).

Small-Animal PET Studies. In vivo studies were conducted under an animal protocol approved by the National Institutes of Health Clinical Center Animal Care and Use Committee. PET scans and their analysis were performed as previously described (4).

Integrin $\alpha_{v} \beta_{3}$-Targeted Radiotherapy and Response Monitoring. Multiple escalating doses of ${ }^{90} \mathrm{Y}-\mathrm{NMEB}-\mathrm{RGD}$ were tested in U87MG tumor xenografts when the tumor volume reached $150 \mathrm{~mm}^{3}$. Mice were divided into 6 groups (5-8 mice per group): group A received saline, group B 
received 7.4 MBq of ${ }^{90} \mathrm{Y}-\mathrm{NMEB}-\mathrm{RGD}$, group C received 3.7 MBq of ${ }^{90} \mathrm{Y}-$ NMEB-RGD, group D received $1.75 \mathrm{MBq}$ of ${ }^{90} \mathrm{Y}-\mathrm{NMEB}-\mathrm{RGD}$, group $\mathrm{E}$ received 7.4 MBq of ${ }^{90} \mathrm{Y}-\mathrm{RGD}$, and group $\mathrm{F}$ received $1.85 \mathrm{MBq}$ of ${ }^{90} \mathrm{Y}-$ RGD. Each animal received an intravenous dose of ${ }^{90}$ Y-NMEB-RGD. Groups B to D received an additional dose at day 14 after treatment.

For HT-29 xenografts, 2 groups were compared: control (injected with saline) and treated (injected with $7.4 \mathrm{MBq}$ of ${ }^{90}$ Y-NMEB-RGD). Antitumor efficacy was evaluated by monitoring the tumor volume and body weight every $2 \mathrm{~d}$. Individual tumor size was calculated with the formula (length $[\times]$ width $[\times]$ width)/2. Groups $A, B, D$, and $E$ underwent both ${ }^{18} \mathrm{~F}$-FDG PET imaging 3 and $10 \mathrm{~d}$ after the start of treatment and ${ }^{18} \mathrm{~F}$-FLT PET imaging 5 and $12 \mathrm{~d}$ after therapy. The PET imaging protocol was similar to that previously described (4), except that fasting before ${ }^{18} \mathrm{~F}-\mathrm{FDG}$ PET lasted $4 \mathrm{~h}$.

The endpoint criteria for Kaplan-Meier analysis were a weight loss of more than $15 \%$, a tumor volume of greater than $1,600 \mathrm{~mm}^{3}$, active ulceration of the tumor, and abnormal behavior indicating pain or unease.

Biodistribution. Mice bearing U87MG tumor xenografts injected with ${ }^{64} \mathrm{Cu}$-NMEB-RGD were sacrificed after 24-h time-point PET imaging. Blood, muscle, bone, liver, kidneys, spleen, intestine, heart, and tumor were collected and wet weighed. For mice bearing MDAMB-435 and HT-29 tumor xenografts, blood, tumor, and heart were collected, and radioactivity was assayed with a $\gamma$-counter. The results were expressed as percentage injected dose per gram $(\% \mathrm{ID} / \mathrm{g})$.

\section{RESULTS}

\section{Preparation of Radiolabeled Compounds}

The new EB-RGD derivatives were prepared by linking a truncated EB molecule to a lysine spacer, attaching a chelator to the $\alpha$-amine, attaching maleimide to the $\varepsilon$-amine, and attaching the thiolated RGD peptide to maleimide (Fig. 1). The usage of NOTA or DOTA depended on the radionuclide to be complexed. We prepared 4 compounds as tests and controls for the purposes of ligand validation (Fig. 1; Supplemental Fig. 1) (supplemental materials are available at http://jnm.snmjournals.org): ${ }^{64} \mathrm{Cu}-\mathrm{NMEB}-\mathrm{RGD}$, which has a high affinity for integrin $\alpha_{\mathrm{v}} \beta_{3}$ and contains an albuminbinding moiety; ${ }^{64} \mathrm{Cu}$-NMEB-RAD, a control peptide with no affinity for the integrin $\alpha_{\mathrm{v}} \beta_{3}$ receptor (11) but retaining the albuminbinding moiety; ${ }^{64} \mathrm{Cu}-\mathrm{NOTA}-\mathrm{RGD}$, which has a high affinity for the integrin $\alpha_{\mathrm{v}} \beta_{3}$ receptor but lacks the albumin-binding moiety; and ${ }^{64} \mathrm{Cu}-\mathrm{NEB}$, which is the albumin-binding moiety $(4,12)$.

\section{In Vitro Characterization of NMEB-RGD}

The affinity of NMEB-RGD for binding to integrin $\alpha_{\mathrm{v}} \beta_{3}$ was compared with that of RGD in competition assays with U87MG cells. Preliminary experiments showed that competition with NMEB-RGD in the presence of HSA did not reach equilibrium at $1 \mathrm{~h}$; hence, further experiments were performed at $4 \mathrm{~h}$. In competitions with ${ }^{64} \mathrm{Cu}-\mathrm{RGD}$ (specific activity, $6.6 \mathrm{GBq} / \mu \mathrm{mol}$ ), the $\mathrm{IC}_{50} \mathrm{~S}$ (mean \pm SD) for NMEB-RGD and RGD at $4 \mathrm{~h}$ were $74.1 \pm 28.2$ and $85.2 \pm 14.0 \mathrm{nM}$, respectively (Fig. 2A). The affinity of NMEBRGD for binding to albumin $(2.34 \pm 1.38 \mu \mathrm{M})$ (Fig. 2B) was comparable to the published affinity of EB for binding to albumin $(\sim 2.5 \mu \mathrm{M})(13,14)$.

Changing the chelator from NOTA to DOTA did not change the affinity of binding for integrin $\alpha_{\mathrm{v}} \beta_{3}$. In the absence of HSA, the $\mathrm{IC}_{50}$ for DOTA-RGD was $67.8 \pm 33.6 \mathrm{nM}$, and that for DMEB-RGD was $76.6 \pm 31 \mathrm{nM}$; in the presence of $1 \% \mathrm{HSA}$, the $\mathrm{IC}_{50}$ for DOTA-RGD was $185.23 \pm 121.28 \mathrm{nM}$ (Supplemental Fig. 2A). Replacement of the glycine residue in the RGD peptide with alanine abolished its binding to the receptor (Supplemental Fig. 2B).

\section{Cellular Uptake and Internalization}

${ }^{64} \mathrm{Cu}$-NMEB-RGD cell uptake and inter-
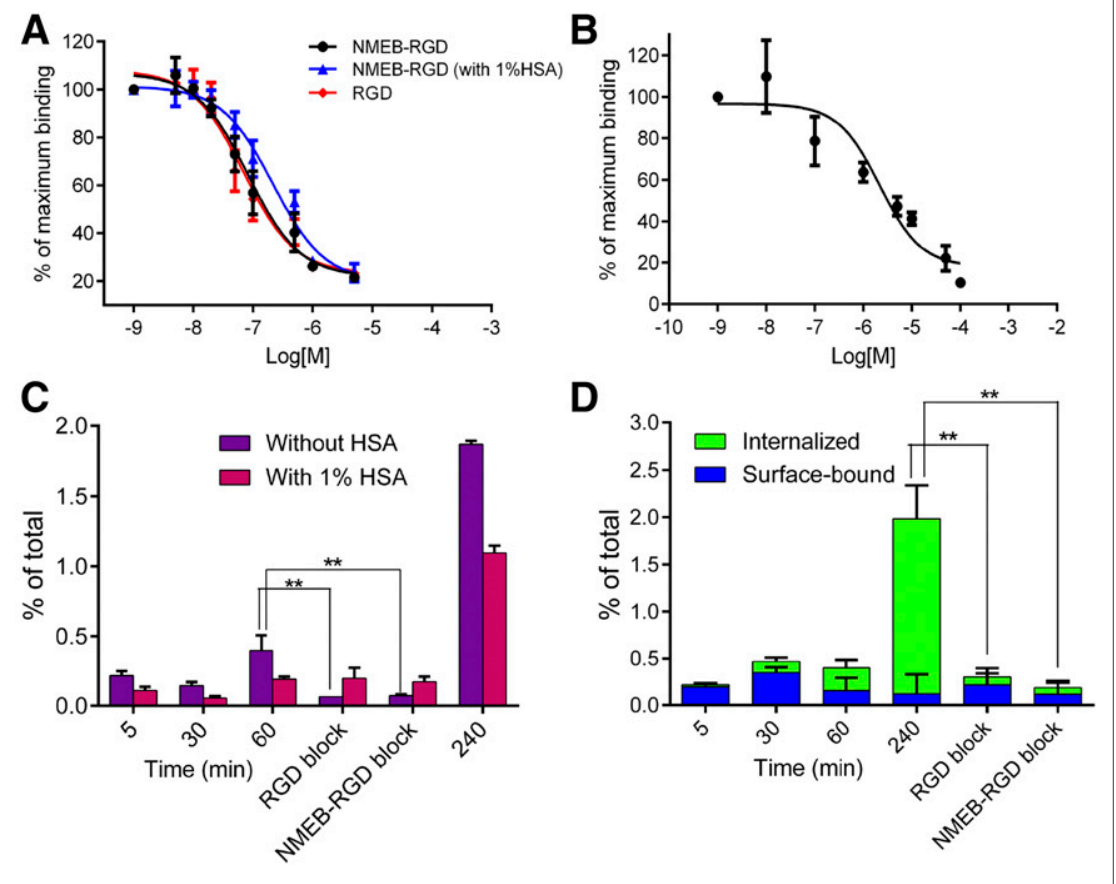

FIGURE 2. (A) Cell-binding assays with U87MG cells, ${ }^{64} \mathrm{Cu}-\mathrm{RGD}$ as competitor, and 4 -h incubation time. (B) Assay of binding of NMEB-RGD to fluorescein isothiocyanate-albumin with avidin beads. (C) Uptake of ${ }^{64} \mathrm{Cu}-\mathrm{NMEB}-\mathrm{RGD}$ in U87MG cells without HSA and with $1 \% \mathrm{HSA}$. Results of blocking studies were evaluated at $1 \mathrm{~h}$. (D) Internalization of ${ }^{64} \mathrm{Cu}-\mathrm{NMEB}-\mathrm{RGD}$ in U87MG cells. Green bars represent internalized ${ }^{6} \mathrm{C} C \mathrm{Cu}-\mathrm{NMEB}-\mathrm{RGD}$, and blue bars represent surface-bound ${ }^{64} \mathrm{Cu}-\mathrm{NMEB}-\mathrm{RGD}$. Results of blocking studies were evaluated at $4 \mathrm{~h}$. ${ }^{* *} P<0.01$. nalization were tested with 3 cell lines (U87MG, MDA-MB-435, and HT-29) that express different levels of integrin $\alpha_{v} \beta_{3}$ (high, medium, and low, respectively) $(15,16) .{ }^{64} \mathrm{Cu}-\mathrm{NMEB}-\mathrm{RGD}$ uptake at all time points was significantly higher without albumin in the medium (Fig. 2C). ${ }^{64} \mathrm{Cu}$-NMEBRGD uptake by U87MG cells increased with longer incubation times, reaching $1.86 \% \pm$ $0.22 \%$ of total input at $4 \mathrm{~h}$. For HT-29 cells, the uptake was significantly lower $(0.76 \% \pm$ $0.03 \%$ ) (Supplemental Fig. 3A). The specificity of ${ }^{64} \mathrm{Cu}-\mathrm{NMEB}-\mathrm{RGD}$ for integrin $\alpha_{\mathrm{v}} \beta_{3}$ was tested by coincubation with an excess amount of either RGD or NMEB-RGD (Fig. 2C). At $5 \mathrm{~min},{ }^{64} \mathrm{Cu}-\mathrm{NMEB}-\mathrm{RGD}$ uptake was not due to internalization. However, at later time points, most of the ${ }^{64} \mathrm{Cu}-\mathrm{NMEB}-$ RGD uptake was internalized (Fig. 2D). This phenomenon was also seen for the MDAMB-435 and HT-29 cell lines (Supplemental Figs. 3A-3C). Internalization of NMEBRGD did not induce internalization of albumin (Supplemental Fig. 3D).

\footnotetext{
Stability of ${ }^{64} \mathrm{Cu}-\mathrm{NMEB}-\mathrm{RGD}$

${ }^{64} \mathrm{Cu}-\mathrm{NMEB}-\mathrm{RGD}$ was stable in mouse serum for up to $24 \mathrm{~h}$ with no significant
} 
demetallization (Supplemental Fig. 4A). In vivo, more than $90 \%$ of the radioactivity was bound to the blood proteins and not extractable. Consistent with this finding, the free fraction in the plasma for ${ }^{64} \mathrm{Cu}-\mathrm{NMEB}-\mathrm{RGD}$ was $4.2 \%$, whereas that for ${ }^{64} \mathrm{Cu}-\mathrm{RGD}$ was $51 \%$. The extracted radioactivity showed ${ }^{64} \mathrm{Cu}-\mathrm{NMEB}-\mathrm{RGD}$, with only a small amount of a more polar component appearing at $4 \mathrm{~h}$ after intravenous administration (Supplemental Fig. 4B).

\section{Small-Animal PET Imaging of Tumor Xenografts with ${ }^{64} \mathrm{Cu}$-NMEB-RGD}

Three tumor models with various degrees of tumor vascularization and different levels of cellular integrin $\alpha_{v} \beta_{3}$ receptor expression were selected for imaging studies. U87MG and HT-29 tumors both showed elevated vascularity, as indicated by high levels of CD31 staining, whereas MDA-MB-435 tumors showed relatively weaker staining (Supplemental Fig. 5A). The level of human integrin $\alpha_{v} \beta_{3}$ was highest in U87MG tumor tissue, followed by MDA-MB-435 and then HT-29, which showed much lower levels (Supplemental Fig. 5A). Murine integrin $\beta_{3}$ (CD61) expression levels were in agreement with CD31 staining, in the order U87MG greater than HT-29 greater than MDA-MB-435 (Supplemental Fig. 5A).

All 4 radiotracers were first evaluated in U87MG xenografts with high levels of integrin $\alpha_{v} \beta_{3}$ expression. ${ }^{64} \mathrm{Cu}-\mathrm{NMEB}-\mathrm{RGD}$ showed significantly higher tumor uptake than all of the other tracers at all time points $(9.87 \pm 1.40,14.09 \pm 1.62$, and $16.64 \pm 1.99 \% \mathrm{ID} / \mathrm{g}$ at 1,4 , and $24 \mathrm{~h}$ after injection, respectively) (Fig. 3). ${ }^{64} \mathrm{Cu}-\mathrm{NMEB}-$ RGD uptake in the blood was relatively high at $1 \mathrm{~h}$ after injection $(9.58 \pm 0.84 \% \mathrm{ID} / \mathrm{g})$ but decreased significantly at 4 and $24 \mathrm{~h}$ after injection, to $5.73 \pm 0.67$ and $2.46 \pm 0.25 \% \mathrm{ID} / \mathrm{g}$, respectively; these values resulted in high tumor uptake and tumor-to-background ratio at $24 \mathrm{~h}$ (Fig. 3; Supplemental Figs. 5B and 5C).

${ }^{64} \mathrm{Cu}-\mathrm{NMEB}-\mathrm{RGD}$ uptake in both MDA-MB-435 and HT-29 xenografts was significantly lower than that in U87MG xenografts

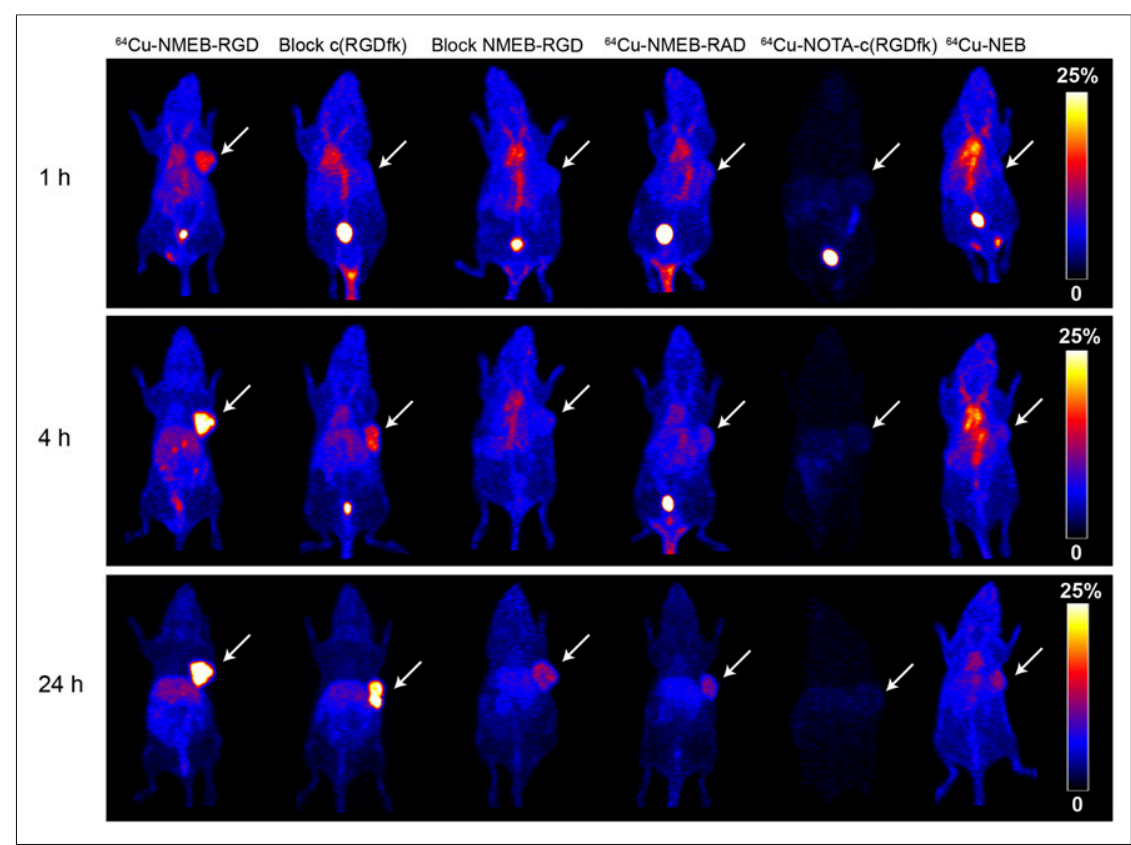

FIGURE 3. Projection PET images of U87MG xenografts at 1, 4, and $24 \mathrm{~h}$ after injection of 3.7 $\mathrm{MBq}$ of ${ }^{64} \mathrm{Cu}-\mathrm{NMEB}-\mathrm{RGD}(n=6),{ }^{64} \mathrm{Cu}-\mathrm{NMEB}-\mathrm{RGD}$ coinjected with $400 \mu \mathrm{g}$ of RGD $(n=4),{ }^{64} \mathrm{Cu}-$ NMEB-RGD coinjected with $400 \mu \mathrm{g}$ of unlabeled NMEB-RGD $(n=3),{ }^{64} \mathrm{Cu}-\mathrm{NMEB}-\operatorname{RAD}(n=4)$, ${ }^{64} \mathrm{Cu}-\mathrm{RGD}(n=4)$, and ${ }^{64} \mathrm{Cu}-\mathrm{NEB}(n=4)$. Arrows indicate tumor location. at all time points (Supplemental Figs. 6A and 6B). When an excess of RGD was coinjected, tumor uptake decreased significantly at $1 \mathrm{~h}$ after injection $(5.77 \pm 0.08 \% \mathrm{ID} / \mathrm{g} ; P=0.017)$. At $4 \mathrm{~h}$ after injection, tumor uptake increased to $11.1 \pm 0.05 \% \mathrm{ID} / \mathrm{g}$; this uptake increased further at $24 \mathrm{~h}$ after injection to values similar to those of ${ }^{64} \mathrm{Cu}-\mathrm{NMEB}-\mathrm{RGD}$ (Fig. 3). Coinjection with an excess amount of unlabeled NMEB-RGD successfully blocked tumor uptake at all time points by 55\%-65\% (Fig. 3; Supplemental Fig. 5C). PET studies were validated by biodistribution at $24 \mathrm{~h}$ (Supplemental Fig. 7A).

As expected, ${ }^{64} \mathrm{Cu}-\mathrm{NOTA}-\mathrm{RGD}$ was rapidly cleared from the blood through the urinary tract and exhibited low accumulation in tumors $(1.29 \pm 0.17,1.15 \pm 0.07$, and $1.06 \pm 0.03 \% \mathrm{ID} / \mathrm{g}$ at 1,4 , and $24 \mathrm{~h}$ after injection, respectively). The nonbinding tracer, ${ }^{64} \mathrm{Cu}-$ NMEB-RAD, had low tumor accumulation (approximately $6 \% \mathrm{ID} / \mathrm{g}$ ) at all time points examined. Its uptake in the blood was significantly higher than that of ${ }^{64} \mathrm{Cu}-\mathrm{NMEB}-\mathrm{RGD}$ at all time points $(10-12 \% \mathrm{ID} /$ $\mathrm{g}$ up to $4 \mathrm{~h}$ after injection and $3.7 \pm 0.3 \% \mathrm{ID} / \mathrm{g}$ at $24 \mathrm{~h}$ after injection). ${ }^{64} \mathrm{Cu}-\mathrm{NEB}$ had the highest accumulation in the blood at all time points, whereas its uptake in tumors was only slightly higher than that of ${ }^{64} \mathrm{Cu}-\mathrm{NMEB}-\mathrm{RAD}(6-7 \% \mathrm{ID} / \mathrm{g}$ up to $4 \mathrm{~h}$ after injection and $\sim 8 \% \mathrm{ID} / \mathrm{g}$ at $24 \mathrm{~h}$ after injection) (Fig. 3; Supplemental Fig. $5 \mathrm{C}$ ).

Uptake in the blood and heart was similar in all 3 models (Supplemental Fig. 7B). To assess the portion of tumor uptake that was due to specific receptor binding, rather than vascularity or an enhanced permeability and retention (EPR) effect, we injected the blood pool imaging tracer, ${ }^{64} \mathrm{Cu}-\mathrm{NEB}$, into MDA-MB-435 and HT29 xenografts. ${ }^{64} \mathrm{Cu}-\mathrm{NEB}$ uptake correlated with CD31 and CD61 staining, in the order U87MG greater than HT-29 greater than MDA-MB-435 (Supplemental Fig. 7C).

\section{${ }^{90}$ Y Radiotherapy}

The unique pharmacokinetics of NMEB-RGD encouraged us to apply this chemical formulation to integrin $\alpha_{\mathrm{v}} \beta_{3}$-targeted radiotherapy. To allow complexation of the radiotherapeutic isotope ${ }^{90} \mathrm{Y}$, the NOTA group of NMEB was replaced with DOTA to produce DMEB. Antitumoral radiotherapy efficacy was evaluated in U87MG tumor-bearing mice treated as indicated in Figure 4A. At day 6 after treatment, significant differences between the groups were evident from the tumor growth curves (Fig. 4B). Tumor volumes from all ${ }^{90}$ Y-DMEB-RGD-treated mice (groups B-D) were significantly lower $(P<0.01)$ than those from the control group (group A) (Fig. 4B). Moreover, starting from day 8 after treatment, significant differences in tumor volumes were observed among all 3 groups of ${ }^{90}$ Y-DMEB-RGDtreated mice and groups $\mathrm{E}$ and $\mathrm{F}$ (Fig. 4B). These differences increased over time, and tumors of mice injected with $7.4 \mathrm{MBq}$ of ${ }^{90} \mathrm{Y}-\mathrm{DMEB}-\mathrm{RGD}$ showed partial regression by day 8 (group B) (Fig. 4B). A second injection of 7.4 MBq of ${ }^{90}$ Y-DMEB-RGD reduced tumor volumes significantly $(P=$ 0.01 at day 20 and $P=0.0003$ at day 22) (Fig. 4B), and tumors almost disappeared by day 30 after the initial treatment.

A minor tumor volume reduction, which lasted for several days after the second 
injection, was observed in group $\mathrm{C}$ (dose, 3.7 MBq), but then tumor volumes increased once more (Fig. 4B). No effect on tumor volumes was observed for group $\mathrm{D}$ (which received a dose of $1.75 \mathrm{MBq}$ ). No systemic toxicity due to radiotherapy was observed by monitoring of animal body weight (Fig. 4C). Kaplan-Meier curves based on the protocol endpoints showed significant differences $(P<0.01)$ between groups B-D and groups A, E, and F. For group B, the survival rate was $100 \%$ up to day 30 after the initial treatment (Fig. 4D).

We tested the therapeutic efficacy of $7.4 \mathrm{MBq}$ of ${ }^{90}$ Y-DMEBRGD in HT-29 mice (Supplemental Figs. 8A and 8B) in comparison with saline-injected controls. The tumor volumes in the treated group and the control group were similar up to $8 \mathrm{~d}$ after treatment, and then the treated tumors started to grow at a slower rate than the control tumors. As a result, ${ }^{90}$ Y-DMEB-RGD-targeted radiotherapy led to a delay in tumor growth in HT-29 tumors, rather than tumor shrinkage, as shown in U87MG tumors (Fig. 4C; Supplemental Fig. 8A).

During treatment, we evaluated tumor metabolism using appropriate PET tracers. ${ }^{18} \mathrm{~F}$-FDG PET imaging $3 \mathrm{~d}$ after injection of ${ }^{90} \mathrm{Y}$ showed decreased uptake, indicating reduced glucose utilization, only for groups injected with ${ }^{90}$ Y-DMEB-RGD (Fig. 5A). However, repeating this scan $10 \mathrm{~d}$ after treatment showed significantly decreased metabolism only for group B (Fig. 5A). ${ }^{18}$ F-FLT PET imaging, an indicator of cellular replication, was conducted 5 and $12 \mathrm{~d}$ after the initial treatment and showed significantly less ${ }^{18} \mathrm{~F}$-FLT uptake for group B (treated with $7.4 \mathrm{MBq}$ of ${ }^{90}$ Y-DMEB-RGD) than for the control group at both imaging times; these results suggested reduced proliferation of tumors in these mice (Fig. 5B).

\section{Tumor Biology After ${ }^{90} \mathrm{Y}$ Radiotherapy}

CD31 staining to evaluate tumor vasculature, Ki-67 staining to evaluate tumor proliferation, and TUNEL staining to determine DNA damage were conducted on tumors excised from all 6 groups (A-F). For groups A, E, and F, with large necrotic areas, live cells on the tumor rim were evaluated. As shown in Figure 5C, the tumor vasculature was lowest in group $\mathrm{B}$ (injected with 7.4 MBq of ${ }^{90}$ Y-DMEB-
RGD). Consistent with ${ }^{18}$ F-FLT PET imaging, a relatively high percentage of cells in groups A and C-F stained positively for $\mathrm{Ki}-67$, whereas significantly reduced cell proliferation was observed in group B (Fig. 5C). Compared with the other 5 groups, group B showed considerably more cell apoptosis, depicted by TUNEL staining (Fig. 5C). Hematoxylin and eosin staining showed that most of the tumor area in group B was already necrotic after treatment with 2 doses of ${ }^{90}$ Y-DMEB-RGD, whereas tumors from the other 5 groups had necrotic areas only in the middle of the tumors; these results suggested that the necrosis was due to insufficient blood supply and hypoxia (Fig. 5C).

\section{DISCUSSION}

One of the major design considerations for a drug is its pharmacokinetics in the blood. A drug with a short half-life in the blood is less available at a target organ. Such a limitation dictates treatment with either high doses or more frequent doses, both of which may increase the likelihood of undesirable side effects. We describe here a novel EB derivative that, on conjugation with therapeutic or targeting agents, results in an enhanced half-life in blood. In addition, the design includes the ability to attach a chelator for radiometals. Therefore, this approach allows the transformation of a drug into a theranostic agent for use as both an imaging agent and a therapeutic agent.

The reversible binding of EB to albumin has been well documented, with a stoichiometry of 8-14 dye molecules per molecule of albumin $(17,18)$. The binding of EB to albumin has been exploited by others to quantify protein leakage as an indicator of increased vascular permeability $(18,19)$. Additionally, Niu et al. used a truncated version of EB to develop a blood volume imaging agent for use in PET (4). We subsequently postulated that the properties of the binding of $\mathrm{EB}$ and its derivatives to albumin can be used to retain drugs or tracers in the blood. We prepared a truncated EB derivative of exendin-4 that proved effective for long-term glucose control (5).

In the present study, we designed a tracer that incorporated an albumin-binding motif based on EB, a chelator for easy labeling with radioactive metal isotopes for imaging or radiotherapy, and a biomolecule-binding motif to provide selectivity for a chosen target. As a proof of concept, we chose the RGD peptide as the targeting biomolecule. This cyclic peptide has been shown to specifically bind integrin $\alpha_{\mathrm{v}} \beta_{3}$ with a high affinity (nM range), rapidly disappear from the blood circulation, and become internalized by receptor-mediated endocytosis $(20,21)$.

Other albumin-binding molecules have been used to modulate pharmacokinetics for therapeutic purposes (14). Trüssel et al. (22) and Dumelin et al. (23) worked extensively to identify albumin-binding molecules by screening chemical libraries; one such molecule was 4-( $p$-iodophenyl)butanoic acid, which was evaluated by Müller et al. for use as a means to improve a folate receptor-binding tracer or radiotherapy agent (24). Subsequent labeling with ${ }^{177} \mathrm{Lu}$ for radiotherapy of tumor-bearing mice produced promising results $(24,25)$. Although
FIGURE 4. (A) Design of therapy protocol. (B) Tumor volume. (C) Body weight change. (D) Survival of mice (5-8/group) injected with saline (group A), $7.4 \mathrm{MBq}$ of ${ }^{90}$ Y-DMEB-RGD (group $\mathrm{B}$ ), $3.7 \mathrm{MBq}$ of ${ }^{90} \mathrm{Y}$-DMEB-RGD (group C), $1.85 \mathrm{MBq}$ of ${ }^{90} \mathrm{Y}$-DMEB-RGD (group D), $7.4 \mathrm{MBq}$ of ${ }^{90} \mathrm{Y}$-DOTA-RGD (group E), and $1.85 \mathrm{MBq}$ of ${ }^{90} \mathrm{Y}-\mathrm{DOTA}-\mathrm{RGD}$ (group $\mathrm{F}$ ) at different days after treatment. Treatment was initiated at day $0 .{ }^{* *} P<0.01$ compared with groups $A, E$, and $F$. 


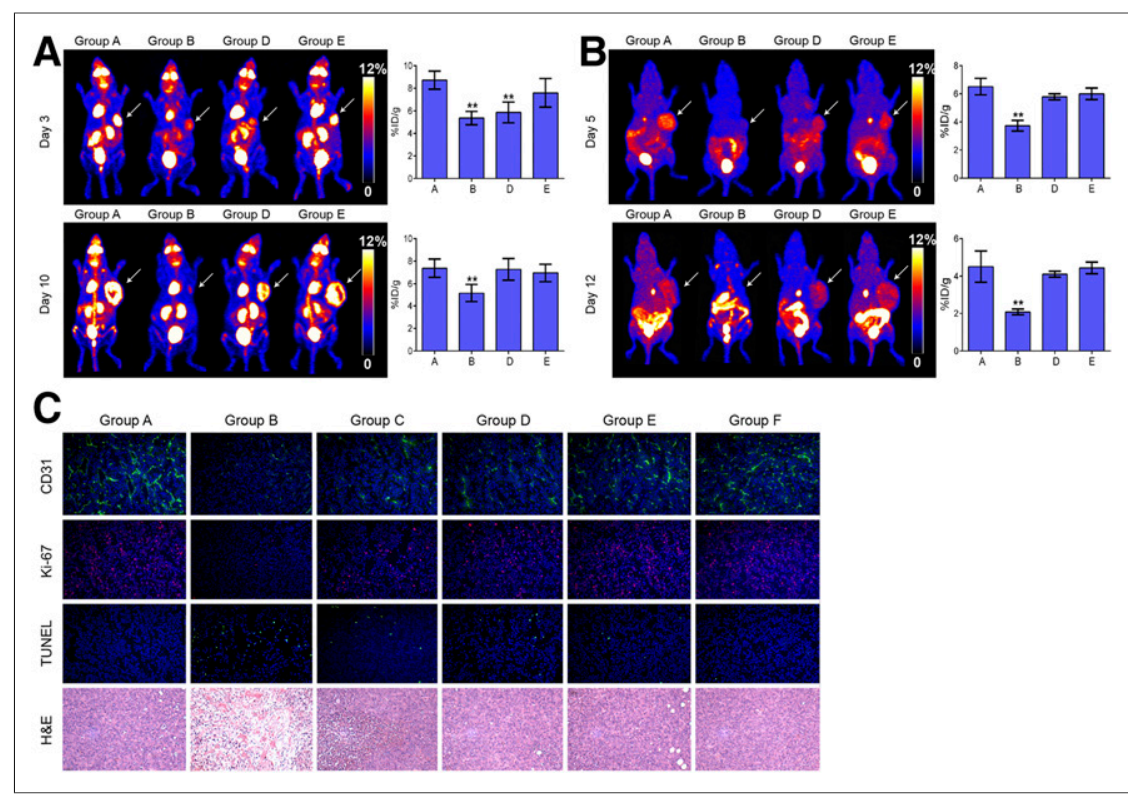

FIGURE 5. Projection PET images of mice injected with ${ }^{18} \mathrm{~F}-\mathrm{FDG} 3 \mathrm{~d}(\mathrm{~A}$, top) and $10 \mathrm{~d}(\mathrm{~A}$, bottom) and ${ }^{18} \mathrm{~F}-\mathrm{FLT} 5 \mathrm{~d}(\mathrm{~B}$, top) and $12 \mathrm{~d}(\mathrm{~B}$, bottom) after treatment with saline (group $\mathrm{A}), 7.4$ $\mathrm{MBq}$ of ${ }^{90} \mathrm{Y}$-DMEB-RGD (group B), $1.85 \mathrm{MBq}$ of ${ }^{90} \mathrm{Y}$-DMEB-RGD (group D), and 7.4 MBq of ${ }^{90} \mathrm{Y}$ DOTA-RGD (group E). (C) Immunofluorescence staining of excised tumors for CD31 (vascularity), Ki-67 (proliferation), TUNEL (apoptosis), and hematoxylin and eosin after radiotherapy treatment was terminated. ${ }^{*} P<0.01$.

Müller et al. did not explicitly mention that their modifications could be used to label other biomolecules, this use seemed to be chemically feasible.

The hypothesis that albumin can serve as a pool and allow the slow release of NMEB-RGD was first supported in binding assays with integrin $\alpha_{\mathrm{v}} \beta_{3}$-expressing cells. In the absence of albumin in the binding buffer, there was no difference between NMEB-RGD and RGD in apparent affinities for binding to these cells. In the presence of albumin, the time to equilibrium was increased, but binding affinities at equilibrium remained similar for both compounds (Fig. 2A). We proposed a mechanism in which the concentration of free NMEB-RGD is initially reduced because of receptor-mediated internalization but equilibrium is maintained by the slow release of albumin-bound NMEB-RGD. To support this hypothesis, we performed internalization assays and found that most of the ${ }^{64} \mathrm{Cu}-$ NMEB-RGD was internalized, with only a small fraction bound to the surface (Fig. 2D). This mechanism is also supported by the observation that fluorescein isothiocyanate-albumin was not internalized in the presence of NMEB-RGD (Supplemental Fig. 3D).

In vivo experiments with ${ }^{64} \mathrm{Cu}-\mathrm{NMEB}-\mathrm{RGD}$ were used to evaluate specific uptake into tumors. Initially, we used ${ }^{64} \mathrm{Cu}-\mathrm{NEB}$ and ${ }^{64} \mathrm{Cu}$-NMEB-RAD to evaluate the EPR effect. In experiments with ${ }^{64} \mathrm{Cu}$-NMEB-RGD, we found that EPR was responsible for about $35 \%$ of tumor uptake (Fig. 3; Supplemental Fig. 5C). Blocking experiments done with both RGD and NMEB-RGD showed that RGD, which does not bind to albumin, blocked ${ }^{64} \mathrm{Cu}-\mathrm{NMEB}-\mathrm{RGD}$ accumulation in tumors at $1 \mathrm{~h}$. However, because of rapid turnover of the receptor and fast clearance of RGD from the blood, the blocking effect was limited to early time points after injection; in addition, ${ }^{64} \mathrm{Cu}$-NMEB-RGD slowly released from albumin over a longer time period was not blocked. At $24 \mathrm{~h}$, the accumulation of ${ }^{64} \mathrm{Cu}-\mathrm{NMEB}-\mathrm{RGD}$ with RGD blocking was similar to that without RGD blocking (Fig. 3; Supplemental Fig. 5C). On the other hand, blocking with NMEB-RGD successfully inhibited ${ }^{64} \mathrm{Cu}-\mathrm{NMEB}-\mathrm{RGD}$ accumulation in tumors, reducing the observed $\% \mathrm{ID} / \mathrm{g}$ by about $65 \%$.

The residual $35 \%$ was accredited to EPR or other nonspecific uptake mechanisms, consistent with EPR estimates from the nontargeting NMEB-RAD. The 3-fold-higher uptake of ${ }^{64} \mathrm{Cu}-\mathrm{NMEB}-\mathrm{RGD}$ than of ${ }^{64} \mathrm{Cu}-\mathrm{NMEB}-\mathrm{RAD}$ demonstrated the benefit of targeted delivery. The proportion of nonspecific binding will preclude us from using ${ }^{64} \mathrm{Cu}$-NMEB-RGD to quantitatively estimate integrin receptor levels, but it remains useful as an imaging agent for estimating the expected uptake of analogous radiotherapeutics.

The uptake of ${ }^{64} \mathrm{Cu}-\mathrm{NMEB}-\mathrm{RGD}$ by neovasculature can be estimated by comparing uptake in HT-29 tumors (low expression of integrin $\alpha_{\mathrm{v}} \beta_{3}$ ) and uptake in U87MG tumors (high expression of integrin $\alpha_{\mathrm{v}} \beta_{3}$ ). Both tumors have high levels of murine vascular integrin expression. HT-29 tumor uptake was about half U87MG tumor uptake. Because we ascribed $35 \%$ of tumor uptake to the EPR effect, on the basis of observed, non-receptor-mediated NEB uptake, the remaining $15 \%$ of HT-29 uptake may have been due to uptake by the neovasculature. However, these are crude calculations, and further experiments are needed to evaluate the contributions of neovasculature and neoangiogenesis to ${ }^{64} \mathrm{Cu}-\mathrm{NMEB}-\mathrm{RGD}$ uptake.

An important observation from the in vivo experiment was that when we compared ${ }^{64} \mathrm{Cu}-\mathrm{NMEB}-\mathrm{RGD}$ and ${ }^{64} \mathrm{Cu}-\mathrm{RGD}$, we saw about a 10-fold increase in the accumulation of radioactivity in tumors (Supplemental Fig. 5B). This high accumulation is direct proof that the use of the same molar amount of the tracer produced significantly higher accumulation in the target organ, which was our aim. This higher uptake was mediated by slower pharmacokinetics, afforded by albumin binding, and resulted in enhanced radionuclide uptake that provided higher imaging contrast. Moreover, the high accumulation of the tracer in the target organ resulted in enhanced sensitivity, enabling the detection of tumors that expressed lower amounts of integrin and that otherwise might have been detected as negative for integrin $\alpha_{v} \beta_{3}$.

The enhanced uptake observed when ${ }^{64} \mathrm{Cu}-\mathrm{NMEB}-\mathrm{RGD}$ was used for imaging predicted that radiotherapy could be conducted with a suitably prepared analog. We chose to use the therapeutic radionuclide ${ }^{90}$ Y. Because NOTA does not chelate yttrium, the EB derivative was resynthesized with a DOTA chelator for the radiotherapeutic isotope ${ }^{90} \mathrm{Y}$; the resulting analog was denoted as DMEB-RGD. DOTA provides strong and stable chelation for ${ }^{90} \mathrm{Y}(26,27)$ but is not optimal for ${ }^{64} \mathrm{Cu}$.

Previous reports on the use of integrin $\alpha_{\mathrm{v}} \beta_{3}$ as a radiotherapeutic target involved ${ }^{90}$ Y-labeled RGD and labeling of a specific antibody with ${ }^{90} \mathrm{Y}(28-31)$. The results obtained with ${ }^{90} \mathrm{Y}-\mathrm{RGD}$ were not encouraging, perhaps because the peptide was cleared rapidly from the blood and showed very low tumor residence time. However, the long time required for the clearance of antibodies from the blood might result in a high absorbed dose in nontumor tissues.

Tumor-bearing mice were treated with various doses of ${ }^{90} \mathrm{Y}-$ DMEB-RGD on days 0 and 14 . The therapeutic response to the 
radiotherapy was scored on the basis of both survival and changes in tumor volume. At a high dose (7.4 MBq), the tumors shrank to almost unobservable sizes (Fig. 4B). They also showed reduced uptake of ${ }^{18} \mathrm{~F}-\mathrm{FDG}$ and ${ }^{18} \mathrm{~F}$-FLT, suggesting reduced proliferation of tumor cells and reduced metabolism (Figs. 5A and 5B). TUNEL staining confirmed that the tumors in the group that received the highest dose of ${ }^{90}$ Y-DMEB-RGD underwent apoptosis. The therapeutic response was confirmed by immunostaining for CD31 (a vascularization marker) and $\mathrm{Ki}-67$ (a proliferation marker).

\section{CONCLUSION}

We developed a novel molecule containing an albumin-binding motif, a chelator for radiometals, and a point of attachment for drugs or targeting molecules. The chelator was selected on the basis of the desired radiometal, and we used both NOTA and DOTA. This novel molecule, when conjugated with a drug or targeting molecule, transformed the attached compound into a theranostic agent, enabling radiolabeling for imaging and radiotherapy. The major feature was the inclusion of an EB-derived albumin-binding moiety that affected the pharmacokinetics in a positive manner to enhance tumor uptake. The increased uptake provided high contrast for diagnostic imaging purposes and increased radiotherapeutic dose delivery. The results shown here for radiolabeled NMEB-RGD and DMEB-RGD are promising and should be further investigated and confirmed with other therapeutic agents.

\section{DISCLOSURE}

The authors gratefully acknowledge the National Natural Science Foundation of China (81471684 and 81371596) and the Intramural Research Program, National Institute of Biomedical Imaging and Bioengineering, National Institutes of Health (NIH). The NIH, its officers, and its employees do not recommend or endorse any company, product, or service. Haojun Chen was partially funded by the China Scholarship Council (CSC). No other potential conflict of interest relevant to this article was reported.

\section{REFERENCES}

1. Caliceti P, Veronese FM. Pharmacokinetic and biodistribution properties of poly (ethylene glycol)-protein conjugates. Adv Drug Deliv Rev. 2003;55:1261-1277.

2. Pedder SC. Pegylation of interferon alfa: structural and pharmacokinetic properties. Semin Liver Dis. 2003;23(suppl 1):19-22.

3. Zhang F, Liu MR, Wan HT. Discussion about several potential drawbacks of PEGylated therapeutic proteins. Biol Pharm Bull. 2014;37:335-339.

4. Niu G, Lang L, Kiesewetter DO, et al. In vivo labeling of serum albumin for PET. J Nucl Med. 2014;55:1150-1156.

5. Liu Y, Wang G, Zhang H, et al. Stable Evans blue derived exendin-4 peptide for type 2 diabetes treatment. Bioconjug Chem. 2016;27:54-58.

6. Chen H, Wang G, Lang L, et al. Chemical conjugation of Evans blue derivative: a strategy to develop long-acting therapeutics through albumin binding. Theranostics. 2016;6:243-253.

7. Cai W, Niu G, Chen X. Imaging of integrins as biomarkers for tumor angiogenesis. Curr Pharm Des. 2008;14:2943-2973.
8. Haubner R, Maschauer S, Prante O. PET radiopharmaceuticals for imaging integrin expression: tracers in clinical studies and recent developments. Biomed Res Int. 2014;2014:871609.

9. Zhao D, Jin X, Li F, Liang J, Lin Y. Integrin $\alpha_{\mathrm{v}} \beta_{3}$ imaging of radioactive iodinerefractory thyroid cancer using ${ }^{99 \mathrm{~m}}$ Tc-3PRGD2. J Nucl Med. 2012;53:1872-1877.

10. Martin SJ, Eisenbarth JA, Wagner-Utermann U, et al. A new precursor for the radiosynthesis of $\left[{ }^{18}\right.$ F]FLT. Nucl Med Biol. 2002;29:263-273.

11. Yang J, Guo H, Miao Y. Technetium-99m-labeled Arg-Gly-Asp-conjugated alpha-melanocyte stimulating hormone hybrid peptides for human melanoma imaging. Nucl Med Biol. 2010;37:873-883.

12. Zhang J, Lang L, Zhu Z, Li F, Niu G, Chen X. Clinical translation of an albuminbinding PET radiotracer ${ }^{68} \mathrm{Ga}-\mathrm{NEB}$. J Nucl Med. 2015;56:1609-1614.

13. Peters T Jr. All About Albumin: Biochemistry, Genetics, and Medical Applications. San Diego, CA: Academic Press; 1996:76-132.

14. Liu Z, Chen X. Simple bioconjugate chemistry serves great clinical advances: albumin as a versatile platform for diagnosis and precision therapy. Chem Soc Rev. 2016;45:1432-1456.

15. Gianni T, Cerretani A, Dubois R, et al. Herpes simplex virus glycoproteins H/L bind to cells independently of $\alpha_{\mathrm{V}} \beta_{3}$ integrin and inhibit virus entry, and their constitutive expression restricts infection. J Virol. 2010;84:4013-4025.

16. Cai $\mathrm{W}, \mathrm{Wu} \mathrm{Y}$, Chen $\mathrm{K}$, Cao $\mathrm{Q}$, Tice $\mathrm{DA}$, Chen $\mathrm{X}$. In vitro and in vivo characterization of ${ }^{64} \mathrm{Cu}$-labeled Abegrin, a humanized monoclonal antibody against integrin $\alpha_{\mathrm{v}} \beta_{3}$. Cancer Res. 2006;66:9673-9681.

17. Rawson RA. The binding of T-1824 and structurally related diazo dyes by the plasma proteins. Am J Physiol. 1943;138:708-717.

18. Lindner V, Heinle H. Binding properties of circulating Evans blue in rabbits as determined by disc electrophoresis. Atherosclerosis. 1982;43:417-422.

19. Saria A, Lundberg JM. Evans blue fluorescence: quantitative and morphological evaluation of vascular permeability in animal tissues. J Neurosci Methods. 1983; 8:41-49.

20. Garanger E, Boturyn D, Dumy P. Tumor targeting with RGD peptide ligands: design of new molecular conjugates for imaging and therapy of cancers. Anticancer Agents Med Chem. 2007;7:552-558.

21. Sancey L, Garanger E, Foillard S, et al. Clustering and internalization of integrin $\alpha_{v} \beta_{3}$ with a tetrameric RGD-synthetic peptide. Mol Ther. 2009;17:837-843.

22. Trüssel S, Dumelin C, Frey K, Villa A, Buller F, Neri D. New strategy for the extension of the serum half-life of antibody fragments. Bioconjug Chem. 2009;20:2286-2292.

23. Dumelin CE, Trussel S, Buller F, et al. A portable albumin binder from a DNAencoded chemical library. Angew Chem Int Ed Engl. 2008;47:3196-3201.

24. Müller C, Struthers H, Winiger C, Zhernosekov K, Schibli R. DOTA conjugate with an albumin-binding entity enables the first folic acid-targeted ${ }^{177}$ Lu-radionuclide tumor therapy in mice. J Nucl Med. 2013;54:124-131.

25. Haller S, Reber J, Brandt S, et al. Folate receptor-targeted radionuclide therapy: preclinical investigation of anti-tumor effects and potential radionephropathy. Nucl Med Biol. 2015;42:770-779.

26. Boswell CA, Sun X, Niu W, et al. Comparative in vivo stability of copper-64labeled cross-bridged and conventional tetraazamacrocyclic complexes. J Med Chem. 2004;47:1465-1474.

27. Garrison JC, Rold TL, Sieckman GL, et al. In vivo evaluation and small-animal PET/CT of a prostate cancer mouse model using ${ }^{64} \mathrm{Cu}$-bombesin analogs: sideby-side comparison of the CB-TE2A and DOTA chelation systems. J Nucl Med. 2007;48:1327-1337.

28. Liu Z, Shi J, Jia B, et al. Two ${ }^{90}$ Y-labeled multimeric RGD peptides RGD4 and 3PRGD2 for integrin targeted radionuclide therapy. Mol Pharm. 2011;8:591-599.

29. Janssen ML, Oyen WJ, Dijkgraaf I, et al. Tumor targeting with radiolabeled $\alpha_{v} \beta_{3}$ integrin binding peptides in a nude mouse model. Cancer Res. 2002;62:6146-6151.

30. Chen $\mathrm{H}$, Niu G, Wu H, Chen X. Clinical application of radiolabeled RGD peptides for PET imaging of integrin $\alpha_{\mathrm{v}} \beta_{3}$. Theranostics. 2016;6:78-92.

31. Veeravagu A, Liu Z, Niu G, et al. Integrin $\alpha_{\mathrm{v}} \beta_{3}$-targeted radioimmunotherapy of glioblastoma multiforme. Clin Cancer Res. 2008;14:7330-7339. 